\title{
The impact of New Labour's English health inequalities strategy on geographical inequalities in infant mortality: a time-trend analysis
}

\author{
Tomos Robinson, ${ }^{\oplus 1}$ Heather Brown, ${ }_{1}^{1}$ Paul D Norman, ${ }^{02}$ Lorna K Fraser, ${ }^{3}$ Ben Barr, ${ }^{4}$ \\ Clare Bambra ${ }^{1}$
}

- Additional material is published online only. To view please visit the journal online (http://dx.doi.org/10.1136/ jech-2018-211679)

${ }^{1}$ Institute for Health and Society, Newcastle University, Newcastle upon Tyne, UK

${ }^{2}$ School of Geography, University of Leeds, Leeds, UK ${ }^{3}$ Department of Health Sciences, University of York, York, UK ${ }^{4}$ Institute of Psychology Health and Society, University of Liverpool, Liverpool, UK

\section{Correspondence to}

Professor Clare Bambra, Newcastle University Institute for Health and Society, Newcastle upon Tyne NE2 4AX, UK; clare.bambra@ncl.ac.uk

Received 3 October 2018 Revised 16 January 2019 Accepted 14 February 2019 Published Online First 19 March 2019

\section{ABSTRACT}

Background The English health inequalities strategy (1999-2010) aimed to reduce health inequalities between the most deprived local authorities and the rest of England. The multifaceted strategy included increased investment in healthcare, the early years, education and neighbourhood renewal. The objective of this study was to investigate whether the strategy was associated with a reduction in geographical inequalities in the infant mortality rate (IMR).

Methods We used segmented regression analysis to measure inequalities in the IMR between the most deprived local authorities and the rest of England before, during and after the health inequalities strategy period.

Results Before the strategy was implemented (1983-1998), absolute inequalities in the IMR increased between the most deprived local authorities and the rest of England at a rate of 0.034 annually $(95 \% \mathrm{Cl} 0.001$ to 0.067$)$. Once the strategy had been implemented (1999-2010), absolute inequalities decreased at a rate of -0.116 annually $(95 \% \mathrm{Cl}-0.178$ to -0.053$)$. After the strategy period ended (2011-2017), absolute inequalities increased at a rate of 0.042 annually $(95 \% \mathrm{Cl}-0.042$ to 0.125$)$. Relative inequalities also marginally decreased during the strategy period. Conclusion The English health inequalities strategy period was associated with a decline in geographical inequalities in the IMR. This research adds to the evidence base suggesting that the English health inequalities strategy was at least partially effective in reducing health inequalities, and that current austerity policies may undermine these gains.

\section{INTRODUCTION}

The infant mortality rate (IMR), defined as the number of deaths under the age of 1 per 1000 live births, can be seen as a key indicator of the environment in which babies are born ${ }^{1}$ and, more broadly, can serve as a proxy for the health of a population. ${ }^{2}$ After years of steady improvement, the English IMR has begun to increase in recent years. ${ }^{3}$ England, like many other countries, also has substantial inequalities in IMRs according to geographical area and socioeconomic group. ${ }^{4}$ For example, national figures from 2016 published by the Office for National Statistics (ONS) have shown that the IMR was 5.9 per 1000 live births in the most deprived areas and 2.6 in the least deprived areas. $^{3}$
Following the election of the New Labour government in 1997, there was a clear attempt to use government policy levers to reduce health inequalities. Consequently, the UK became the first European country in which policy-makers systematically and explicitly attempted to reduce inequalities in health, focusing specifically on supporting families, engaging communities in tackling deprivation, improving prevention, increasing access to healthcare and tackling the underlying social determinants of health. ${ }^{5}$

This comprehensive, multifaceted programme to reduce health inequalities in England was one of the most ambitious strategies of its kind. For example, the strategy included large increases in levels of public spending on a range of social programmes, the introduction of the national minimum wage, area-based interventions such as the Health Action Zones and a substantial increase in expenditure on the National Health Service (NHS). The latter was targeted at more deprived neighbourhoods when, after 2001, a 'health inequalities weighting' was added to the way in which NHS funds were geographically distributed, so that areas of higher deprivation received more funds per head to reflect higher health need. ${ }^{6}$

Furthermore, the then government made tackling health, social and educational inequalities a public service priority by setting public service agreement targets. The key targets of the Labour government's health inequalities strategy were to (1) reduce the relative gap in life expectancy at birth between the most deprived local authorities and the English average by $10 \%$ by 2010 , and (2) cut relative inequalities by occupational class in the IMR by $10 \%$. However, with a change in government and the establishment of the ConservativeLiberal Democrat coalition in 2010, this strategy came to an end. The change of government also led to an introduction of austerity measures intended to reduce the national deficit. These measures were realised through a structured and significant reduction in levels of public spending, including substantial cuts to funding for local authorities, real term reductions to the NHS budget, cuts to the education sector and various reductions across the welfare system. ${ }^{7}$ The scale of the cut is typified by the closure of as many as 1000 Sure Start children's centres since $2010 .^{8}$

A number of studies have subsequently attempted to evaluate whether the English health inequalities 
strategy was a success or not. Overall, the findings are mixed. Prominent early studies ${ }^{57}$ concluded that although the strategy can be considered a partial success, it failed to reach its ambitious targets. However, these studies were published shortly after the end of the strategy period (2010-2011), by which time it is unlikely that the impact had been fully realised. The studies also could not take into account the trend in inequality after the strategy period had ended.

A more recent study $^{10}$ used a modified difference-in-difference approach to compare trends in health inequalities in England both over time and between other European countries. The study found no detectable effect of the English health inequalities strategy on health inequalities, as compared with trends in Finland, the Netherlands and Italy. However, although the empirical studies mentioned above and others ${ }^{11-13}$ found no effect or increases in inequalities during the strategy period, other studies have found that social inequalities in unemployment, child poverty, housing quality, educational attainment and mortality amenable to healthcare decreased during the strategy period. $^{14-17}$

Most recently, Barr et al ${ }^{18}$ have shown using time trend regression analysis that geographical health inequalities marginally decreased during the English health inequalities strategy period, reversing a previously increasing trend. There was also evidence that inequalities had started to increase again following the end of the strategy period. However, these previous studies have largely focused on the first part of the health inequalities strategy, which was aimed at reducing inequalities in life expectancy. No previous study has instead examined the effect of the strategy on its second aim-to reduce inequalities in the IMR. We therefore investigated whether the English health inequalities strategy was associated with a decrease in inequalities in the IMR between the most disadvantaged local authorities and the rest of England, compared with trends both before and after the strategy period.

\section{METHODS \\ Data}

We used data from the UK Data Archive and the ONS on the annual number of births and infant deaths in local authority (LA) areas across England between 1983 and 2017. These data were mapped to 323 lower tier local authorities, excluding the Isles of Scilly, City of London and Rutland due to their small population sizes. The outcome variable, the IMR, was calculated as the ratio of number of deaths under the age of 1 per LA per year to the number of live births per LA per year, multiplied by $1,000{ }^{19}$

We used the Townsend index of material deprivation to identify the most deprived local authorities in England. ${ }^{20}$ This census-based index is made up of four variables (unemployment, non-car ownership, non-home ownership and household overcrowding), which can be used to generate a total score for a particular geographical area. All input data were adjusted to the current LA boundaries. ${ }^{21}$ This measure has previously been used in a variety of settings, including as an input to resource allocation mechanisms and as a measure of deprivation in academic studies. $^{22}$ Although the original New Labour health inequalities strategy target was to reduce the gap in the IMR 'between the routine and manual groups and the population as a whole', analysing this gap is not possible with the data available to us. In this study, we have taken a geographical approach, as this is the best available proxy, and also one which fits within the broad aims of the health inequalities strategy.

The strategy was introduced to bring those from the lowest socioeconomic groups to the same level as the rest of the country. Therefore, in the main analysis, our measure of deprivation was a dummy variable indicating whether the LA area was in the bottom deprivation quintile. The quintiles were population weighted such that $20 \%$ of the population were in each category. We used the Townsend index from 1991 in the main analysis to represent the situation before the start of the strategy period. We also checked the robustness of the results to deprivation measured in each of the 1981, 2001 and 2011 Censuses, and an alternative measure of deprivation-the 2004 Index of Multiple Deprivation. These results are shown in online supplementary appendix 1 .

\section{Empirical analyses}

The analyses focused on comparing changes in the IMR between the most deprived local authorities and the rest of England. A priori, we expected the level of inequality to decrease during the health inequalities strategy period. To estimate the changes in the level of absolute inequality over time, we estimated fixed effects (FE) segmented linear regression models, including marginal spline terms to take account of the time trends, with breakpoints at the beginning and end of the health inequalities strategy period. We interacted these time trends with a dummy variable indicating the deprived group of local authorities, allowing the change in the IMR to vary by the level of deprivation. This segmented regression model therefore provided an estimate of the trend in the absolute difference in the IMR between the most deprived local authorities and the rest of England before, during and after the health inequalities strategy period. The full model specification is presented in online supplementary appendix 2 .

\section{Robustness tests}

To fully assess the robustness of our findings, we subjected our empirical analysis to a large number of robustness tests. We estimated models using different breakpoints to the main analysis to ensure that our results were not driven by our a priori choice (online supplementary appendix 3). We estimated random effects (RE) models rather than FE models (online supplementary appendix 4). We included linear spline terms rather than marginal spline terms (online supplementary appendix 5). To account for outliers, we estimated models with the top 1\%, top $5 \%$, bottom $1 \%$ and bottom $5 \%$ of IMR observations trimmed, respectively (online supplementary appendix 6). Rather than FE models with the IMR in each local authority area as the dependent variable, we estimated Poisson regression models using the number of deaths in each local authority area per year as the dependent variable and the number of births in each local authority area as the offsetting variable (online supplementary appendix 7). We used different specifications of deprivation, specifically a categorical measure comparing the 5 quintiles of deprivation (online supplementary appendix 8) and a continuous measure of deprivation (online supplementary appendix 9). Finally, we included a measure of ethnicity in the RE model (online supplementary appendix 10), as it has previously been shown that ethnicity may be a significant risk factor for infant mortality. ${ }^{23}$ Ethnicity measures could not be included in the FE models, as data were not available in a consistent manner across all census years.

\section{RESULTS}

IMR trends for those in the most deprived local authorities and the rest of England are shown graphically in figure 1. For both the most deprived local authorities and the rest of England, there has been a decline in the IMR over time. For the most deprived 


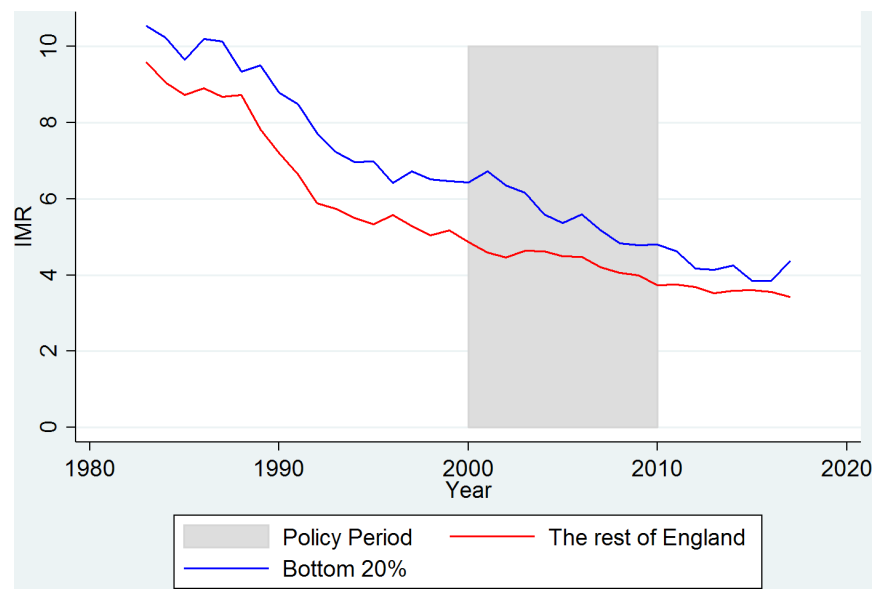

Figure 1 Trends in the infant mortality rate (IMR) in the most deprived local authorities and the rest of England.

local authorities, the average IMR has decreased from 10.53 deaths per 1000 live births in 1983 to 4.36 deaths per 1000 live births in 2017, while for the rest of England this rate has decreased from 9.59 deaths per 1000 live births in 1983 to 3.43 deaths per 1000 live births in 2017.

Trends in the absolute and relative gap between the most deprived areas and the rest of England are shown graphically in figures 2 and 3, respectively. Before the strategy had been implemented (1983-1999), absolute inequality in the IMR between the most deprived areas and the rest of England had gradually increased from 0.95 in 1983 to 1.28 in 1999 . Once the strategy had been implemented (2000-2010), there was evidence of a decrease in absolute inequalities in the IMR, from 1.57 in 2000 to 1.06 in 2010. After the strategy period had ended 20112017), absolute inequalities increased from 0.87 in 2011 to 0.93 in 2017.

There was also evidence of a very small decrease in relative inequalities during the strategy period. Before the start of the strategy period, the relative gap in the IMR between the deprived local authorities and the rest of England increased from 1.10 in 1983 to 1.25 in 1999 . Once the strategy had been implemented, the relative gap in the IMR decreased marginally, from 1.32 in 2000 to 1.29 in 2010 . After the strategy period had ended, the relative gap in the IMR marginally increased from 1.23 in 2011 to 1.27 in 2017.

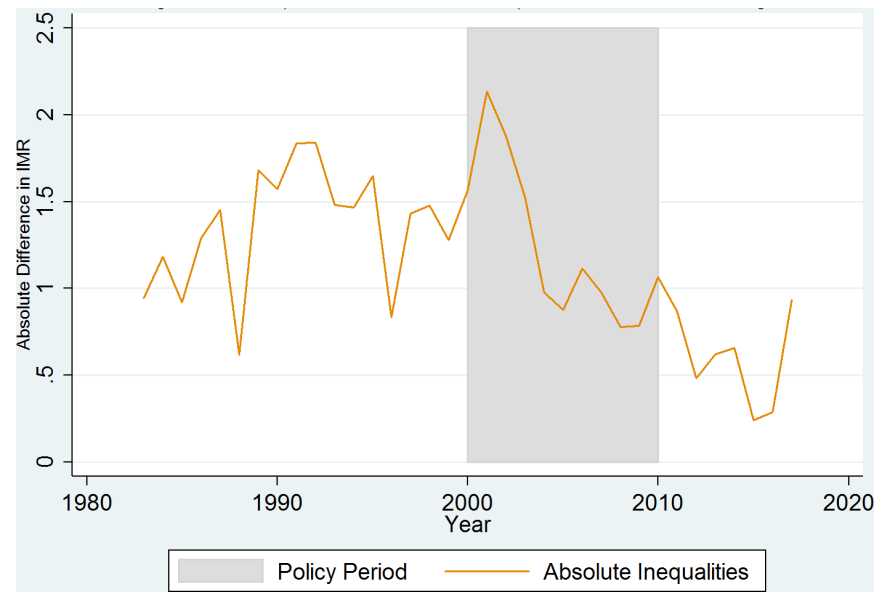

Figure 2 Absolute inequalities in the infant mortality rate (IMR) between the most deprived local authorities and the rest of England.

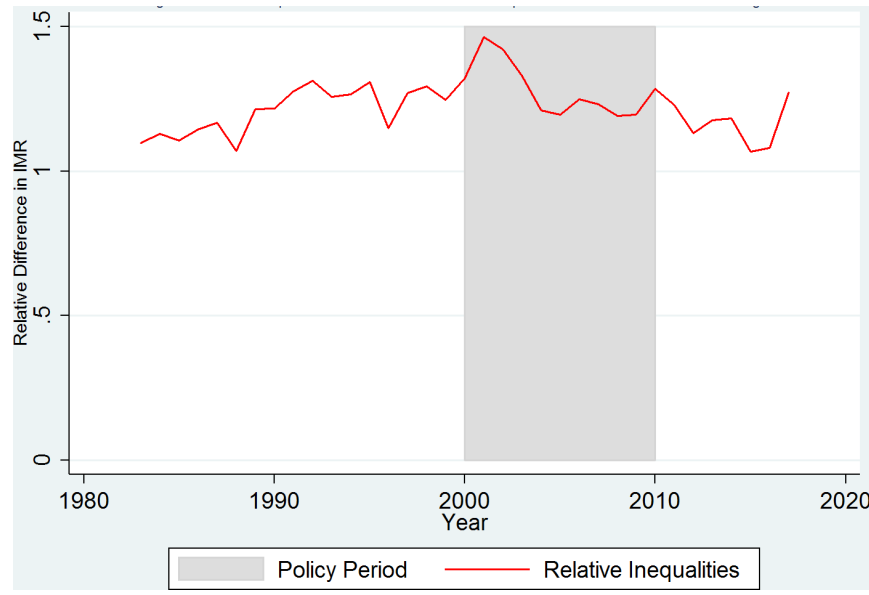

Figure 3 Relative inequalities in the infant mortality rate (IMR) between the most deprived local authorities and the rest of England. Estimates based on a fixed-effects regression model using local authority panel dataset of IMR from 1983 to 2017. Break points at 1999 and 2010.

The results from our preferred FE segmented regression model (table 1) show the trend in absolute infant mortality inequalities estimated before, during and after the strategy period. Before the strategy period, the absolute gap in the IMR between the most deprived local authorities and the rest of England increased at an average of 0.034 per year (95\% CI 0.001 to 0.067 ). During the strategy period, the gap in the IMR between the most deprived local authorities and the rest of England decreased at an average rate of $-0.116(95 \% \mathrm{CI}-0.178$ to -0.053$)$ per year. After the strategy period, the gap in the IMR increased at an average rate of 0.042 ; however, these estimates were not statistically precise (95\% CI -0.042 to 0.125 ).

The various robustness checks we implemented allowed us to explore the level of uncertainty around the estimates of the trends in absolute inequalities before, during and after the strategy period. In FE models using a binary measure of deprivation, the lower bound of the trend before the strategy period was 0.016 (95\% CI -0.017 to 0.049 ) (online supplementary appendix 6D) and the upper bound was 0.053 (95\% CI 0.008 to 0.097 ) (online supplementary appendix 1B). During the strategy period, the lower bound of the trend was $-0.075(95 \%$ $\mathrm{CI}-0.109$ to -0.042 ) (online supplementary appendix 6B) and the upper bound was $-0.187(95 \% \mathrm{CI}-0.268$ to -0.106$)$ (online supplementary appendix 1B). After the strategy period, the lower bound of the trend was -0.040 (95\% CI -0.100 to

Table 1 Trend in absolute inequalities in the infant mortality rate (IMR) between the most deprived local authorities and the rest of England, before, during and after the English $\mathrm{HI}$ strategy period

\begin{tabular}{|c|c|}
\hline Period & $\begin{array}{l}\text { Annual change in absolute gap in the IMR between the } \\
\text { most deprived local authorities and the rest of England } \\
(95 \% \mathrm{CI})\end{array}$ \\
\hline Before 1983-1999 & $0.034(0.001$ to 0.067$)$ \\
\hline During 2000-2010 & $-0.116(-0.178$ to -0.053$)$ \\
\hline After 2011-2017 & $0.042(-0.042$ to 0.125$)$ \\
\hline \multicolumn{2}{|c|}{$\begin{array}{l}\mathrm{N}=11305 \text { ( } 323 \text { local authorities } \times 35 \text { years), overall } \mathrm{R}^{2}=0.3857 \text {. } \\
\text { Trend is shown as the annual increase or decrease (minus values) in the absolute gap in } \\
\text { the IMR (defined as the number of deaths under } 1 \text { per } 1000 \text { births in the local authority } \\
\text { area). }\end{array}$} \\
\hline
\end{tabular}


0.021 ) (online supplementary appendix 5) and the upper bound was 0.097 (95\% CI -0.026 to 0.220 ) (online supplementary appendix 1B). It should also be noted that varying the break points specified in the main analysis made relatively little difference to the empirical results (online supplementary appendix 3).

\section{DISCUSSION}

Our study suggests that geographical inequalities in infant mortality declined in both absolute and relative terms during the English health inequalities strategy period, a time in which the government specifically introduced policies aimed at reducing socioeconomic inequalities in infant mortality. Prior to the strategy being implemented, there was evidence of gradually increasing geographical inequalities in both absolute and relative terms. There was no conclusive evidence that the reduction in geographical inequalities has continued after the strategy period.

The results from this study differ from several previous studies which have shown that the English health inequalities strategy did not fully meet its ambitions. ${ }^{5}{ }^{7-13}$ Instead, our study is more in line with the recent work of Barr et al, ${ }^{18}$ which found that socioeconomic inequalities in both male and female life expectancy decreased during the English health inequalities strategy period, and have also begun to increase again after the strategy period. The results are also in line with relatively recent evidence from the USA, ${ }^{24}$ which has shown that when taking account of the overall downward trend in infant mortality, the IMR is an average of 3\% lower during Democratic administrations compared with Republican administrations. Democrats, like New Labour in the 2000s, are more likely to expand public expenditure on healthcare and welfare, while Republicans have tended to decrease expenditure in these areas. Other research from the USA has found that welfare and public health expansion in the 1960s and 1970s following the civil rights acts led to reductions in inequalities in IMR between African Americans and White Americans. ${ }^{25}$

Although our analysis suggests that there was a decrease in the level of inequality in the IMR during the English health inequalities strategy period, we cannot conclusively say that this is causal relationship, and there are other factors that may explain the decrease in absolute inequality during this period. For example, for a substantial duration of the strategy period (up to the 2008 financial crash), the UK encountered relative macroeconomic stability, with annual growth in GDP and lower rates of unemployment. Recent research from the USA has argued that there may be a significant relationship between macroeconomic conditions and infant mortality, mediated through factors such as the availability of good quality prenatal care. ${ }^{26}$ Indeed, infant mortality can be seen to be directly caused by a number of factors, including immaturity, congenital abnormalities, intrapartum causes and sudden infant deaths, ${ }^{27}$ which in turn have a number of complex and interacting risk factors, including smoking, ${ }^{28}$ maternal obesity in pregnancy, ${ }^{29}$ maternal age ${ }^{11}$ and ethnicity. ${ }^{23}$ Although some of these risk factors are modifiable (smoking and obesity) and may be affected by healthcare, public health and social policy, others are not.

There are several strengths in our analysis. First, we have analysed changes over time within local authorities, which, unlike cross-sectional analysis, enabled us to control for time constant differences between local authorities. Second, we conducted an extensive range of robustness checks to check that our main conclusions were not driven by the model specification used in the main analysis. However, despite these advantages, we must note some important limitations. First, we were not able to establish a true causal relationship between the English health inequalities strategy and IMR. For example, it may be the case that the observed decrease in inequalities in the IMR was due to a broader government strategy rather than the health inequalities strategy itself. Second, as with the majority of research in this subject area, our analysis used area-based measures, which are potentially subject to the 'ecological fallacy', which assumes that all individuals residing in a certain geographical area have similar characteristics. Third, we were unable to differentiate neonatal and postneonatal deaths. Our results also may have differed if we had used infant survival rather than infant mortality as our outcome measure ${ }^{30}$ or occupational class instead of an area measure of deprivation. However, births and deaths data by occupational class were not available to us with appropriate time increments to assess the English health inequalities strategy. Finally, as we were only able to measure the level of deprivation every 10 years (in line with the national Census), regression to the mean may have impacted our results.

The results from this study also have important implications for current health policy, especially given the recent increase in infant mortality in England in recent years. Since 2010, successive Conservative-led English governments have introduced a programme of austerity. This has included substantial cuts to funding for local authorities, real terms reductions to the NHS budget, cuts to the education sector and various reductions across the welfare system. Our analysis suggests that it is increases in public spending on healthcare and welfare that are associated with decreases in inequalities in the IMR, and this is something that should be learnt from by current and future governments. Current government policies are arguably going in the wrong direction and may squander some of the gains made in the health inequalities strategy period.

Finally, although the reduction in relative inequalities during the policy period was shown to be very small in magnitude, it has been argued by a number of prominent health inequalities researchers that an overall decrease in the level of mortality will inevitably result in an increase in relative inequalities, ${ }^{31}$ and therefore any policy intervention that either maintains or

\section{What is already known on this subject?}

- Implemented between 1999 and 2010, the English health inequalities strategy was one of the most ambitious government strategies of its kind, resulting in large increases in levels of public spending on a range of social programmes including a substantial increase in expenditure on the National Health Service.

- Since it came to an end, a number of studies have attempted to evaluate whether the English health inequalities strategy was a success or not. The results thus far are mixed.

\section{What does this study add?}

- In this study, we add to the empirical literature by analysing the association between the health inequalities strategy and both absolute and relative inequalities in the infant mortality rate, using subnational data from 323 local authorities and segmented regression analysis.

- Our results suggest that absolute and relative inequalities reduced during the strategy period. 
decreases the level of relative inequality can be considered at least a partial success. However, it has also been argued that implementing interventions that decrease absolute inequalities but do not substantially decrease relative inequalities may result in groups at the lower end of the social hierarchy at a significant comparative disadvantage. As argued in the Marmot Review, ${ }^{11}$ focusing solely on the most disadvantaged in society 'will not reduce health inequalities sufficiently'. This implies that in order to reduce health inequalities in health (including inequalities in infant mortality), population-wide strategies should be used in combination with evidence-based interventions specifically targeted at those areas of higher deprivation that have a higher health need: with increased levels of infant mortality: a proportionate universal approach.

\section{CONCLUSION}

The multifaceted English health inequalities strategy, implemented between 1999 and 2010, was associated with a decrease in geographical inequalities in the IMR between the most and less deprived English local authorities. These results imply that government policies specifically introduced to decrease inequalities in health may be beneficial, and that their discontinuation as a result of austerity may see inequalities increasing again.

Contributors TR conducted the empirical analysis and led the drafting and revising of the manuscript with support from $\mathrm{HB}$ and $\mathrm{CB}$. HB designed the analytical strategy, led data interpretation and substantially supported the drafting and revising of the manuscript. HB is the guarantor of the analysis. PDN provided the data, contributed to data interpretation and revisions of the manuscript. LKF contributed to data interpretation and commented on manuscript drafts. BB advised on the analytical strategy and data interpretation. CB is the corresponding author and the guarantor of the study. She planned the study, contributed to analytical design, data interpretation and substantially supported the drafting and revising of the manuscript. All authors agreed the final version.

Funding This study is funded by a Leverhulme Trust Research Leadership Award (reference RL-2012-006), awarded to CB. CB and HB are also members of Fuse, the Centre for Translational Research in Public Health (www.fuse.ac.uk). Fuse is a UK Clinical Research Collaboration (UKCRC) Public Health Research Centre of Excellence. Funding for Fuse from the British Heart Foundation, Cancer Research UK, Economic and Social Research Council, Medical Research Council, the National Institute for Health Research, under the auspices of the UKCRC, is gratefully acknowledged. Grant reference number is MR/K02325X/1.

Disclaimer The views expressed in this paper do not necessarily represent those of the funders, the Leverhulme Trust or UKCRC. The funders had no role in study design, data collection and analysis, decision to publish or preparation of the manuscript.

Competing interests None declared.

Patient consent for publication Not required.

Provenance and peer review Not commissioned; externally peer reviewed.

Data sharing statement The statistical code is available from the lead author on request (tom.robinson@ncl.ac.uk).

\section{REFERENCES}

1 Macfarlane A, Mugford M. Birth counts. Vol. 1. Statistics of pregnancy and childbirth. Vol. 2. Statistics of pregnancy and childbirth: tables. HMSO 1984.

2 Reidpath DD, Allotey P. Infant mortality rate as an indicator of population health. J Epidemiol Community Health 2003;57:344-6.

3 Office for National Statistics. Child mortality in England and Wales: 2016. Available: https://www.ons.gov.uk/peoplepopulationandcommunity/birthsdeathsandmarriages/ deaths/bulletins/childhoodinfantandperinatalmortalityinenglandandwales/2016 [Accessed 24 Jul 2018].

4 Norman P, Gregory I, Dorling D, et al. Geographical trends in infant mortality: England and Wales, 1970-2006. Health Stat Q 2008;1.

5 Mackenbach JP. The English strategy to reduce health inequalities. Lancet 2011:377:1986-8.

6 Bambra C. Health divides: where you live can kill you. Policy Press, 2016.

7 Mackenbach JP. Can we reduce health inequalities? An analysis of the English strategy (1997-2010). J Epidemiol Community Health 2011;65:568-75.

8 The Sutton Trust. 1,000 children's centres 'lost' since 2009, 2009. Available: https:// www.suttontrust.com/newsarchive/1000-childrens-centres-closed-since-2009/ [Accessed 10 Sept 2018].

9 Mackenbach JP. Has the English strategy to reduce health inequalities failed? Soc Sci Med 2010;71:1249-53.

$10 \mathrm{Hu} \mathrm{Y}$, van Lenthe FJ, Judge $\mathrm{K}$, et al. Did the English strategy reduce inequalities in health? A difference-in-difference analysis comparing England with three other European countries. BMC Public Health 2016;16.

11 Marmot M, Allen J, Goldblatt P, et al. Fair society, healthy lives. The Marmot Review. 2010.

12 Department of Health. Tackling health inequalities: 2007 status report of the program for action. London, 2007. Available: http://webarchive.nationalarchives.gov.uk/+/ www.dh.gov.uk/en/Publicationsandstatistics/Publications/DH_083471 [Accessed 24 Jul 2018].

13 Department of Health. Tackling health inequalities: 10 years on. London, 2009. Available: http://webarchive.nationalarchives.gov.uk/20130107105354/http:/www. dh.gov.uk/prod_consum_dh/groups/dh_digitalassets/documents/digitalasset/dh_ 098934.pdf [Accessed 24 Jul 2018].

14 Bambra C. Reducing health inequalities: new data suggest that the English strategy was partially successful: table 1. J Epidemiol Community Health 2012;66:662.1-662.

15 Barr B, Bambra C, Whitehead M. The impact of NHS resource allocation policy on health inequalities in England 2001-11: longitudinal ecological study. BMJ 2014;348:93231.

16 Buck D, Dixon A. Improving the allocation of health resources in England. London: Kings Fund, 2013.

17 Asaria M, Ali S, Doran T, et al. How a universal health system reduces inequalities: lessons from England. J Epidemiol Community Health 2016;70:637-43.

18 Barr B, Higgerson J, Whitehead M. Investigating the impact of the English health inequalities strategy: time trend analysis. BMJ 2017;26.

19 Handbook of Vital Statistics Systems and Methods, Volume 1: Legal, Organisationaland Technical Aspects, United Nations Studies in Methods, Glossary, Series F, No. 35. New York, United Nations, 1991.

20 Townsend P, Phillimore P, Beattie A. Health and deprivation: inequality and the North. Routledge, 1988.

21 Norman PD, Darlington-Pollock F. The changing geography of deprivation in Great Britain: exploiting Small Area census data. 1971 to 2011.

22 Senior M. Deprivation indicators. the census data system 2002:123-39.

23 Moser K, Stanfield KM, Leon DA. Birthweight and gestational age by ethnic group, England and Wales 2005: introducing new data on births. Health Stat $Q$ 2008;39:22-31.

24 Rodriguez JM, Bound J, Geronimus AT. US infant mortality and the President's party. Int J Epidemiol 2014;43:818-26.

25 Krieger N, Chen JT, Coull B, et al. The unique impact of abolition of Jim Crow laws on reducing inequities in infant death rates and implications for choice of comparison groups in analyzing societal determinants of health. Am J Public Health 2013;103:2234-44.

26 Orsini C, Avendano M. Macro-economic conditions and infant health: a changing relationship for black and white infants in the United States. PLoS One 2015; 10:e0123501.

27 Gray R, Hollowell J, Brocklehurst P, et al. Health inequalities infant mortality target: technical background. Inequalities in infant mortality project briefing paper 2009;2.

28 Johansson AL, Dickman PW, Kramer MS, et al. Maternal smoking and infant mortality: does quitting smoking reduce the risk of infant death? Epidemiology 2009;1:590-7.

29 Meehan S, Beck CR, Mair-Jenkins J, et al. Maternal obesity and infant mortality: a meta-analysis. Pediatrics 2014;133:863-71.

30 Kjellsson G, Gerdtham U-G, Petrie D. Lies, damned lies, and health inequality measurements: understanding the value judgments. Epidemiology 2015;26.

31 Scanlan JP. Race and mortality. Society 2000;37:29-35. 